\title{
BIBLIOGRAPHIE
}

des ouvrages consultés au cours du présent travail.

H. T. Converse. Journal of Dairy Science, V IX, no 4, p. 388. Effets, sur la production laitière, de rations plus fortes que celles indiquées par les normes d'Haecker, d'Eckles et de Savage.

C. H. Eckles et T. W. Gullickson. Journal of Agricultural Research, vol, 42, $\mathrm{n}^{\circ}$, Washington, May 1931, p. 603-616. Besoins nutritifs pour la croissance normale des vaches laitières.

B. Forbes. Journal of Dairy Science, V IX, no 4, p. 373. Besoins d'énergie pour les vaches laitières,

A.-M. Lmroy. Comptes rendus du Congrès de l' Alimentation du Bétail et du Contrốle laitier. Société Nationale d'Encouragement à l'Agriculture. Paris, 1925. Nouvelles étud əs sur la valeur pratique des tables d'alimentation pour vaches laitières.

A.-M. LERoy. Revue de Zootechnie, n० 2-3, février-mars 1933. Conseils aux éleveurs de vaches laitières.

Mergs et Converse. Journal of Dairy Science, V, vol. VIII, n ${ }^{\text {os }} 5$ et 11, p. 177 et 523. Besoins d'énergie des vaches laitières.

Stations de recherches danoises :

Bulletin 136, 1931. Influence du taux de protéine de la ration sur la production laitière.

Bulletin 144, 1932. Recherches sur l'alimentation des vaches laitières avec betteraves fourragères, demi-sucrières et sucrières.

\section{LA PASTEURISATION DU LAIT EN VUE DE LA FABRICATION DES FROMAGES (1)}

\author{
par JEAN PIEN
}

- Docteur ès Sciences, Ingénieur chimiste (I. C. R.)

Directeur des Laboratoires des "Fermiers Réunis"

L'une des grandes préoceupations actuelles des techniciens du lait et des hygiénistes, est la pasteurisation obligatoire du lait.

La preuve est faite des dangers dn lait cru et des bienfaits de la pasteurisation. Tout récemment encore, au XIVe Congrès de Chimie Industrielle (Octobre 1934), nous avons rappelé et développé les raisons pour lesquelles le lait eru est ou peut être un danger, et nous avons également apporté les preuves de l'efficacité de la pasteurisation.

Ces raisons ont tellement paru convaincantes, qu'un vœu tendant à rendre obligatoire la pasteurisation du lait, a été adopté à l'unanimité.

Nous pouvons d'ailleurs dire que la question est à l'étude dans

(1) Extrait du Bulletin de l'Association des Chimistes de Sucrerie, Distillerie et Indrustrie Agricoles (156, boulevard Magenta) ; numéro de décembre 1934. 
les sphères officielles, et nous croyons pouvoir affirmer que dans peu de mois, la question aura été résolue dans le sens souhaité par les hygiénistes, en général, et par les pédiâtres, en particulier.$$
*^{*} *
$$

Le point de vue qui domine toutes ces études et tous ces débats, est incontestablement celui de l'alimentation de l'enfant dans l'allaitement artificiel.

Est-ce à dire qu'il faille négliger le souei de l'amélioration de la qualité du lait pour l'adulte ? Certes non. Et c'est pour répondreà ce souci respectable de généralisation, que de nombreux hygiénistes préconisent, non seulement la pasteurisation obligatoire pour les laits de consommation en nature, mais encore celle des laits destinés à l'extraction đe la crème (done à la préparation du beurre) et à la fabrication du fromage.

Il est certain que la logique, autant que l'hygiène, semble commander la pasteurisation de tous ces laits; et les efforts que nous faisons pour aider les hygiénistes à triompher dans cette bienfaisante campagne sociale qu'ils mènent en faveur de la pasteurisation obligatoire du lait de vente en nature, ne devraient pas diminuer quand il s'agit de la question des dérivés du lait.

En fait, nous n'hésitons pas à écrire que cette question nouvelle est toute différente de la précédente et beaucoup plus complexe.

La pasteurisation du lait destiné à être transformé en crème, beurre, fromages, soulève des problèmes techniques d'une difficulté insoupçonnable et des problèmes économiques d'une importance capitale.

Ces divers points n'ont pour ainsi dire jamais été soulevés. On s'est contenté jusqu'à présent, de dire à ceux qui, comme nous, préconisent la pasteurisation du lait de consommation en nature : "Vous avez raison de désirer l'assainissement du lait de vente directe. Mais il faut aller jusqu'au bout dans le progrès et pasteuriser le lait destiné à la fabrication du beurre et du fromage. "

Jusqu'alors, aucun technicien du lait n'a répondu à l'hygiéniste en lui montrant les dangers techniques d'une généralisation aussi hâtive et, disons le mot, aussi prématurée. Nous voulons précisément essayer de jeter dans la discussion des éléments nouveaux qui nous sont très familiers; la position que nous avons personnellement prise dans la question de la pasteurisation du lait nous donne, plus qu'à tout autre, le droit de dire jusqu'où on peut aller dans cette voie, et quels sont les domaines qui nous paraissent, momentanément du moins, interdits à la pasteurisation.

$$
*^{*} *
$$

Le fait fondamental est le suivant : la fabrication de certains 
fromages paraît actuellement irréalisable, en faisant appel aux techniques classiques, si on utilise du lait pasteurisé.

On sait que le point capital de la fabrication d'un fromage est l'égouttage du caillé, e'est-à-đire l'élimination đu sérum après l'emprésurage. Dans le cas du fromage à égouttage provoqué (fromages pressés ou cuits), on parvient dans certains cas à reproduire parfaitement, avec du lait pasteurisé réensemencé de ferments lactiques purs, la marche qualitative et quantitative de l'égouttage des fromages de lait cru; de ce fait, on peut arriver à obtenir par cette technique des fromages aussi bons et même quelquefois meilleurs que ceux qui proviennent de lait non pasteurisé.

Mais dans le cas de fromages à égouttage spontané (fromages à pâte molle du type camembert ou brie), la constitution physicochimique du lait est altérée par la pasteurisation de manière telle, que les conditions de l'égouttage sont complètement modifiées. L'addition de sels de calcium solubles pour améliorer l'action de la présure, l'addition de ferments lactiques pour remplacer ceux qui sont détruits, ne permettent pas d'obtenir un égouttage normal si on travaille dans les conditions habituelles de la fabrication classique.

En fait, et sans qu'il soit possible, ici, d'entrer plus avant dans le détail de cette question, on peut affirmer que la fabrication d'un camembert véritable, d'un brie véritable (avec leurs qualités propres), est pratiquement impossible en partant de lait pasteurisé et en suivant les techniques classiques de fabrication.

Les raisons de cet état de choses ne sont pas toujours connues.

Il faut bien reconnaître que l'édifice moléculaire du complexe phosphocaséinate calcique est modifié par l'action de la chaleur. Il faut reconnaître aussi que la pasteurisation détruit certaines diastases, qui, si elles sont vraisemblablement de peu d'intérêt au point de vue alimentaire, doivent jouer un rôle important dans l'affinage du fromage. Il faut reconnaître encore que la pasteurisation détruit beaucoup plus de germes (qualitativement) qu'on n'en réintroduit ensuite : l'ensemencement du lait pasteurisé se fait presque toujours exclusivement avec des ferments lactiques; il va de soi que le lait cru contenait bien autre chose; et parmi tous les germes que l'on détruit par la pasteurisation, certains ont peut-être à jouer dans la maturation du fromage, un rôle utile, sinon nécessaire, à côté des ferments lactiques.

On peut donc craindre que, dans le cas de certains fromages pour le moins, l'égouttage et la maturation soient des phénomènes moins simples que l'on incline généralement à le croire et qu'il faille, pour en expliquer et en reproduire le mécanisme exact, faire appel à bien d'autres éléments qu'aux seuls ferments lactiques artificiels travaillant sur un édifice moléculaire déjà modifié par la chaleur. 
Si cette hypothèse semble contredite par les sucè̀s certains et parfois éclatants obtenus dans la fabrication de tel ou tel fromage à partir du lait pasteurisé, elle semble, au contraire, renforcée par les échecs, non moins réels, essuyés par la plupart de ceux qui ont essayé de résoudre le problème dans le cas de fromages à pâte molle.

Quand nous parlons d'échecs, nous englobons les demi-suecès enregistrés ici et là - car, en matière de fromagerie, un demi-succès technique est un échec économique. Que l'on se propose de reproduire à l'aide de lait pasteurisé les excellents bries ou camemberts de notre marché français et qu'on parvienne à en faire des fromages neutres, sans finesse, trop standardisés, nous concluons à un échec. Industriellement parlant, la fabrication du camembert ou du brie en lait pasteurisé sera au point (et ce sera alors un gros succès) le jour où l'on sera parvenu à reproduire, et à reproduire à coup sûr, les remarquables produits que certains de nos grands fromagers obtiennent assez régulièrement à l'heure actuelle à partir du lait cru.

En Amérique, où l'on essaie également de fabriquer des fromages à égouttage spontané avec du lait pasteurisé, on déclare que cette opération ne conduit jamais à de meilleurs produits que dans le cas de la fabrieation en lait cru; on estime que la pasteurisation doit être réservée aux mauvais laits, qui seraient incapables de conduire à de bons fromages. Pour les fromagers américains, la pasteurisation du lait destiné aux pâtes molles, ne constitue pas une amélioration de eas favorables, mais seulement un remède aux cas défavorables.

En résumé, dans l'état actuel de nos connaissances, la pasteurisation du lait destiné à la fabrication de certains fromages (à égouttage spontané), ne conđuit pas encore au résultat eherché, tant s'en faut. Et imposer actuellement la pasteurisation du lait pour tous les fromages français conduirait à une catastrophe économique.

Mais nous n'avons nullement l'intention de prétendre condamner à priori cette technique, encore moins de faire une déclaration d'agnosticisme.

Il est possible et souhaitable que les recherches systématiques entreprises dans ce domaine, parviennent un jour à fournir une solution parfaite au problème de la pasteurisation en fromagerie.

Nous voulons, ici, simplement insister sur cette idée, qu'en l'état actuel des choses, il serait prématuré đ'imposer la pasteurisation pour la fabrieation de tous les fromages, car on commettrait une faute technique et économique des plus graves.

De même qu'il eût été utopique de réclamer la pasteurisation obligatoire du lait de vente il y a 30 ans, parce que les techniques de pasteurisation n'étaient pas généralisables, de même, il est actuelle- 
ment impossible, techniquement et économiquement, d'assurer la fabrication de tous les fromages en partant du lait pasteurisé.

En d'autres termes, d'accord sur le principe, nous demandons seulement un long délai pour son étude et son application.

\section{***}

Mais, dira-t-on, puisque les dangers du lait cru sont manifestement établis, ne commettrait-on pas une grosse faute en différant l'obligation de la pasteurisation pour les fromages? Ne doit-on pas, au nom de l'hygiène, imposer dès maintenant la pasteurisation en fromagerie, même au risque de faire baisser la qualité de certains fromages et même au risque de faire disparâ̂tre du marché certains fromages d'une importance économique et nationale très notable, certes, mais moins précieuse, cependant, que les exigences de l'hygiène?

La question est sérieuse, car il est manifeste que le souci de l'hygiène passera toujours les intérêts économiques dans l'esprit du législateur et dans l'opinion publique.

Il ne faut donc pas répondre à la légère à cette question, car elle va se poser dans les mois à venir.

Empressons-nous d'abord de dire, que ce qui est vrai du danger du lait cru à consommer en nature, ne l'est pas d'une manière aussi absolue d'un grand nombre de dérivés du lait, surtout de ceux qui ont été le siège d'une fermentation lactique au cours de leur fabrication.

On sait, en effet, que la fermentation lactique s'oppose dans une large mesure, à la prolifération et à la vie même de certains microbes pathogènes, comme ceux du groupe coli-typhique, qui sont particulièrement sensibles à l'acidité. (Nos travaux personnels ont permis de montrer par exemple, que, dans le lait, la prolifération du $B$. coli est totalement arrêtée par 4 grammes d'acide lactique au litre.)

L'acidification d'un milieu réalise toujours une antisepsie partielle pour de nombreux germes non entraînés à vivre et à se multiplier à des $p H$ très différents de 7 .

Or, dans la maturation des crèmes en vue de la fabrication du beurre, l'acidification atteint souvent et dépasse même quelquefois 4 grammes par litre.

Dans la fabrication des fromages à pâte molle, à égouttage spontané, l'acidification du milieu se poursuit très rapidement pendant cet égouttage et atteint des valeurs beaucoup plus élevées encore. On peut dire que dans le cas du camembert, du brie, du coulommiers, etc., l'égouttage constitue une sorte de pasteurisation chimique partielle qui est sûrement fatale à de nombreux germes pathogènes pouvant se rencontrer dans le lait cru. 
Dans les fromages à pâte pressée, c'est-à-dire à égouttage provoqué, la marche de l'acidification est différente, puisque, dans un grand nombre de ces fromages, le lait est à une acidité relativement faible lors de l'emprésurage (rarement supérieure à 1 gramme d'acide lactique par litre); mais les ferments lactiques se développent. néanmoins dans le caillé pendant la maturation et on sait également. que les ferments lactiques ont, vis-à-vis de certains microbes pathogènes, une action inhibitrice, même en l'absence d'acidité, par suite d'une concurrence vitale, mal connue encore dans son mécanisme, mais certaine et démontrée en fait.

Dans les fromages à pâte cuite, l'usage des températures relativement élevées (de l'ordre de 50 à $55^{\circ}$ ) pendant un temps assez long contribue, lui aussi, à côté de l'action acidifiante et inhibitrice des ferments lactiques, à assainir le milieu.

Cet argument de l'amélioration bactériologique du lait cru (par destruction ou arrêt du développement de certains microbes pathogènes), a reçu des consécrations expérimentales directes très démonstratives, qu'il serait trop long d'énumérer aujourd'hui, mais sur lesquelles nous nous proposons de revenir.

En admettant même qu'il ne faille pas pousser cet argument à l'extrême (car il est possible que certains germes pathogènes puissent traverser toute la vie du fromage sans avoir été détruits), on peut néanmoins affirmer, sur la foi de démonstrations expérimentales sérieuses et impartiales, que :

La fermentation lactique - acte essentiel de la fabrication d'un grand nombre de dérivés du lait (crèmes, beurre, fromages) possède une action antiseptique démontrée vis-à-vis de certains germes pathogènes.

En d'autres termes et comme conclusion de cette première argumentation, un lait cru contenant certains microbes pathogènes définis, peut fort bien conduire à une crème, à un beurre, à des fromages partiellement ou même totalement assainis par l'action combinée de l'acidité des ferments lactiques, du salage, de la température de cuisson du caillé, etc.

A ce premier argument, qui possède une valeur scientifique certaine, s'en ajoute un second, dont l'importance et l'évidence sauteront aux yeux.

Les dangers du lait cru, avons-nous toujours dit (et c'est l'avis de tous les hygiénistes), intéressent surtout l'enfant et prineipalement le nourrisson. S'il est exact que certaines épidémies de fièvre typhoïde d'origine lactée (lait cru) ont fait des victimes chez les adultes, il est encore plus certain que les victimes du lait cru les plus nombreuses (et de beaucoup!) sont les jeunes enfants. Il suffit de relire les. 
fameuses statistiques anglaises de Stanley GRIFFITH, et allemandes de Kummer, pour en être convaincu.

Il y a deux raisons à cet état de choses : d'abord, l'adulte consomme (toute proportion gardée) moins de lait cru que l'enfant, mais surtout, l'enfant est plus vulnérable. L'adulte, lui, possède des résistances, voire même une immunité acquise, qui souvent le protègent contre les dangers des aliments malsains, tel que le lait oru peut l'être, et le protégeraient de la même façon contre les dangers éventuels de fromages malsains, s'il s'en trouvait.

Or, il n'est pas question de faire consommer des fromages à de jeunes enfants, encore moins à des nourrissons.

Plus le danger est grand, e'est-à-dire plus l'enfant est jeune, moins il y a de chances de voir le fromage entrer dans sa ration.

Par conséquent, en admettant que le premier argument soit sans valeur (ce qui va à l'encontre des certitudes acquises), on conçoit que le mal serait, en fait, conjuré pour les êtres les plus vulnérables.

Un troisième argument peut encore être mis en avant, qui concerne plus spécialement le cas de la tuberculose.

Nous venons de dire que l'enfant échapperait au danger du fromage pathogène, s'il existait, pour la bonne raison qu'il n'en consomme pas.

Mais l'adolescent, l'adulte, possèdent-ils des "résistances " suffisantes pour s'opposer à l'infection par le bacille tuberculeux ?

Au point où on en est actuellement de la connaissance de la fuberculose, il semble acquis que la tuberculose de l'adulte n'est presque jamais le résultat d'une infection récente. Tout être humain s'infecterait, plus ou moins, dès son jeune âge - (on a même prétendu que tout adulte bien portant réagissait positivement à la tuberculine). Mais le mal ne se déclarerait vraiment qu'à l'occasion d'une manifestation de moindre résistance, ayant d'ailleurs son origine, plus dans l'état physiologique habituel ou antérieur (ou ancestral) de l'individu que dans son état physiologique du moment. Autrement dit, la tuberculose serait à peu près toujours en puissance ; mais elle ne se développerait que dans certaines circonstances favorables et sur certains terrains préparés. Cette théorie diminuerait donc singulièrement le rôle de la contagion de la tuberculose, et il importerait assez peu, si cette théorie est exacte, qu'un adulte ingérât des aliments apportant des germes tuberculeux.

De toute façon, que ces vues soient exactes ou non, il reste certain que l'enfant est vulnérable, que son alimentation lactée doit être irréprochable, et que le danger éventuel de la consommation de fromage préparé à partir du lait cru, n'existe pas pour lui, puisqu'il n'en consomme pas. 
Pour l'adulte, si la théorie précédente est exacte, le danger éventuel d'un fromage infecté serait négligeable, et si la théorie est inexacte, on peut raisonnablement admettre que le fromage, déjà assaini par sa fabrication même, ne présenterait pas les dangers du lait cru.

$$
*^{*} *
$$

En résumé, les idées que l'on peut, selon nous, équitablement soutenir en matière de pasteurisation du lait destiné à être transformé en fromage, seraient les suivants :

$1^{\circ} \mathrm{Il}$ est conforme à la logique et au souci de l'hygiène, de désirer la pasteurisation de tout lait, quel qu'il soit, même s'il est destiné à la fabrication du fromage.

$2^{\circ}$ Dans l'état actuel de nos connaissances, la fabrication de certains fromages est pratiquement impossible en partant de laits pasteurisés.

$3^{\circ}$ Ces fabrications deviendront peut-être possibles un jour, à la suite de recherches nombreuses et approfondies, mais on ne peut actuellement prévoir la durée de ces recherches, ni la réponse qu'elles apporteront.

$4^{\circ}$ Il faut done absolument maintenir le statu quo pour ce qui est du lait destiné à la fabrication des fromages et n'imposer aucune pasteurisation dans aucune de ces industries, sous peine de voir certaines d'entre elles disparaître immédiatement.

$5^{\circ}$ Il est d'ailleurs avéré que la consommation des fromages fabriqués avec du lait cru, ne présente pas les dangers du lait cru : d'abord parce que la fabrication elle-même réalise un assainissement certain et démontré, ensuite parce que l'enfant, à l'âge où il est le plus vulnérable des consommateurs de lait, ne consomme pas de fromage.

\section{BIBLIOGRAPHIE ANALYTIQUE}

\section{LES LIVRES}

Pien (J.), Martin (R.) et Bergier (M.). - Examen chimique de

la qualité des caséines lactiques. Brochure de 70 pages, 1 figure et nombreux tableaux. - Editée par Le Lait, 16, rue Spontini, Paris (XVIe), 1934. Prix : 20 francs.

Nous sommes très heureux de présenter cette brochure qui réunit des articles parus successivement dans la Revue Le Lait, articles qui constituaient différents chapitres d'une même étude. L'intérêt d'un semblable travail a pu déjà être apprécié. Tous ceux qui éprouveront le besoin, au cours de leurs recherehes de laboratoire ou industrielles, de se documenter sur divers points concernant l'examen chimique de la qualité des caséines 